\title{
Evaluation on Safety Performance of Power Transmission Iron Tower Based on Stress Monitoring
}

\author{
Jiceng Han ${ }^{1}$, Hongjie Zhang ${ }^{2}$, Deyuan Lin ${ }^{1}$, Lanxi Weng ${ }^{3}$, Fei Wang ${ }^{2}$ \\ ${ }^{1}$ Electric Power Research Institute of Fujian Electric Power Company, Fuzhou, 350000 \\ ${ }^{2}$ China Electric Power Research Institute, Beijing, 100055 \\ ${ }^{3}$ Power China Fujian Electric Power Engineering Co., Ltd., Fuzhou, 350000
}

\begin{abstract}
The on-line monitoring of power transmission line mainly refers to the observation of load parameters such as wind speed and ice coating thickness, and not many researches about monitoring and safety status are conducted directly targeting their stress states; however, through when calculating the stress status of structure via load, the simplified assumptions in calculation model and load model introduce some errors. This thesis realized real-time monitoring to stress states of main stress poles of iron tower based on the 36 strain monitoring points distributed on the power transmission iron tower; in addition, based on the analysis on monitoring data, this thesis evaluated the safe using performances of the iron tower.
\end{abstract}

\section{Introduction}

The traditional main on-line monitoring objects include wind speed, equivalent icing thickness, image/video, pole tower inclination, aeolian vibration, conductor temperature, conductor sag, circuit windage yaw, conductor galloping, field pollution grade, etc. From the perspective of monitoring items, the monitoring are mainly conducted targeting external load of power transmission circuit pole tower[1-4]. In recent years, along with the constantly increased attention of power grid to the essential safety of iron tower, the observation only to external load of iron tower cannot meet requirements for high reliability performance index any longer, and the power grid system inner has gradually started the research on monitoring of in-service stress of iron tower, and has obtained some achievements[5]. Huang Xinbo[6] and Ji Xiangke[7] have implemented monitoring to the stress of power transmission iron tower based on the strain measurement technology of optical fiber, and has obtained relatively good test data. However, the monitoring item is single, and there is still some technical difficulty in large-scale promotion and marketization. In addition, the modulator $\&$ demodulator required by the optical fiber type strain gauge is expensive, with low survival rate of optical fiber, not convenient for large-scale application of stress monitoring of iron tower. Zhang Xuan[8] has monitored the iron tower stress with vibrating wire strain gauge, and relatively speaking, the price of resistance type strain gauge is lower, and it also can be installed on the surface of steel structure conveniently, and will not damage the iron tower members[9-12].

Therefore, in this thesis, the No. 52 tower of the coastal Longhou Line-II of Fujian Province was selected as the monitoring object. Firstly, the distribution situation of pole stress of iron tower under the gale working condition was analyzed. Through analysis, a pole with relatively high stress was selected, and then the strain gauge was provided on it for stress monitoring. According to the stress level actually measured during the period of transit of strong breeze, this thesis conducted evaluation on safety performance of iron tower.

\section{Analysis on Finite Element Simulation of Stress Status and Layout of Measuring Points of Iron Tower}

The No. 52 tower of coastal Longhou Line No.-II of Fujian Province was adopted as monitoring object, and then a model for analysis on finite element simulation of iron tower was established. Under the working condition of gale when the design wind speed is $35 \mathrm{~m} / \mathrm{s}$, the stress situation of iron tower pole was calculated. The distribution situation of stress ratio of iron tower pole is as shown in Figure 1. According to the sort descending, the top 18 poles with relatively high stresses were taken out as measuring points to arrange poles (see Table 1). This tower refers to angle steel tower, and all poles refer to angle steel. The two legs of angle steel were arranged with 2 measuring points, and totaling 36 measuring points were arranged along with the whole tower. The numbers of measuring points are as shown in Figure 2. 
GT $99 \%$

$95 \%-99 \%$

$70 \%-85 \%$

LT $70 \%$



Figure 1. Cloud Chart of Calculation Results of Stress Ratio of Iron Tower Poles

Table 1. Top 18 Poles with Relatively High Stress

\begin{tabular}{|c|c|c|c|c|c|}
\hline Sorting & No. of Pole & Stress Ratio & Sorting & No. of Pole & Stress Ratio \\
\hline 1 & $50-210$ & 1.09 & 10 & $1030-1070$ & 0.92 \\
\hline 2 & $170-372$ & 1.09 & 11 & $670-690$ & 0.92 \\
\hline 3 & $530-690$ & 0.99 & 12 & $870-972$ & 0.92 \\
\hline 4 & $350-390$ & 0.98 & 13 & $690-752$ & 0.9 \\
\hline 5 & $210-350$ & 0.97 & 14 & $10-52$ & 0.9 \\
\hline 6 & $10-50$ & 0.93 & 15 & $210-230$ & 0.89 \\
\hline 7 & $772-870$ & 0.93 & 16 & $1171-1211$ & 0.89 \\
\hline 8 & $690-730$ & 0.93 & 17 & $50-230$ & 0.88 \\
\hline 9 & $970-1032$ & 0.93 & 18 & $750-812$ & 0.88 \\
\hline
\end{tabular}




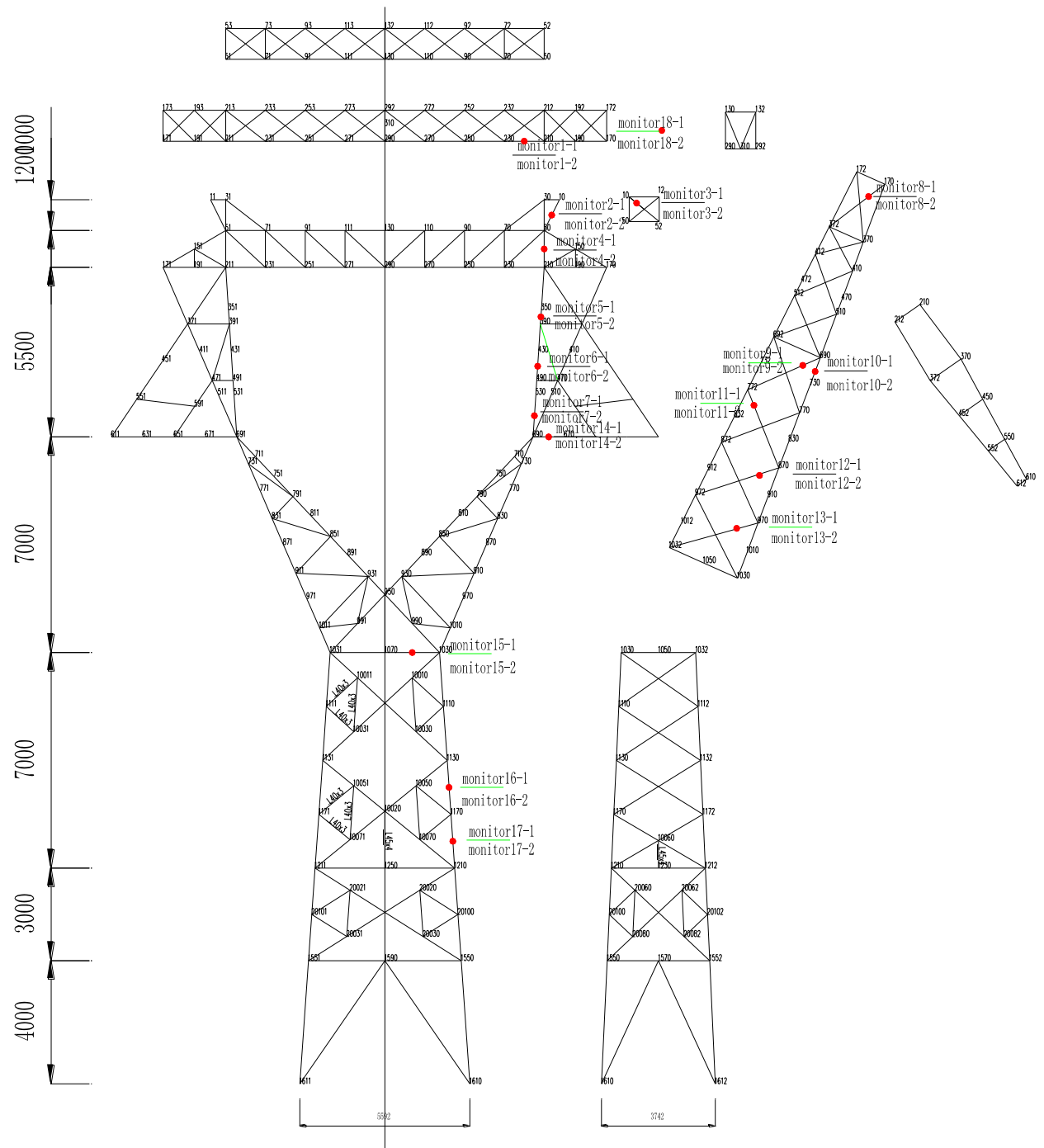

Figure 2. Diagram of Positions of 36 Measuring Points Distributed along with Tower Body

\section{Field Installation of Stress Monitoring System of Iron Tower}

The core part of resistance type strain gauge refers to the wound resistance wire, and the strain gauge clings on the surface of part; if the part deforms due to strain, for the diameter of resistance wire becomes small, their resistance value $\mathrm{R}$ will be changed, and the strain testing will be completed according to the change situation of resistance value measured by electrical bridge; the resistance-strain effect refers to the physical phenomenon that the resistance of metal conductor is deformed (lengthened or shortened) when the conductor suffers from external force, thus causing change. If the metal resistance wire suffers from axial tension, its length will be increased, while its cross section will be decreased, and this will cause increasing of resistance value. On the contrary, when it suffers from axial pressure load, the cross section of resistance wire will be increased, and the resistance value will be decreased. The resistance strain gauge, the elasticity sensitive element and the compensating resistance constitute a kind of multipurpose resistance strain sensor. This thesis adopted the full-bridge circuit as the internal circuit structure of strain gauge, and through calibration, this thesis removed the influence of initial resistance on strain measurement. In this testing, the aerospace-level strain testing sensor was adopted, featured by small temperature drifting $\left(1 \mathrm{PPM} /{ }^{\circ} \mathrm{C}\right)$, long service life (able to be used for 1 year normally in the environment of $-50^{\circ} \mathrm{C}$ to $60^{\circ} \mathrm{C}$ ), and high strain identification sensitivity (the minimum strain identification grade is $0.2 \mu \mathrm{m} / \mathrm{m}$ ). Although the tower has been installed with temperature sensor, and the strain caused by temperature can be eliminated, considering that the drifting caused by the temperature of such sensor is very small, the influence of temperature change on strain testing result is not severe. With regard to the data of 36 measuring points, the monitoring data is transmitted every 10 minutes, and the data communication is transmitted with $4 \mathrm{G}$ network; the data of vibration acceleration sensor is transmitted to the appointed email. 


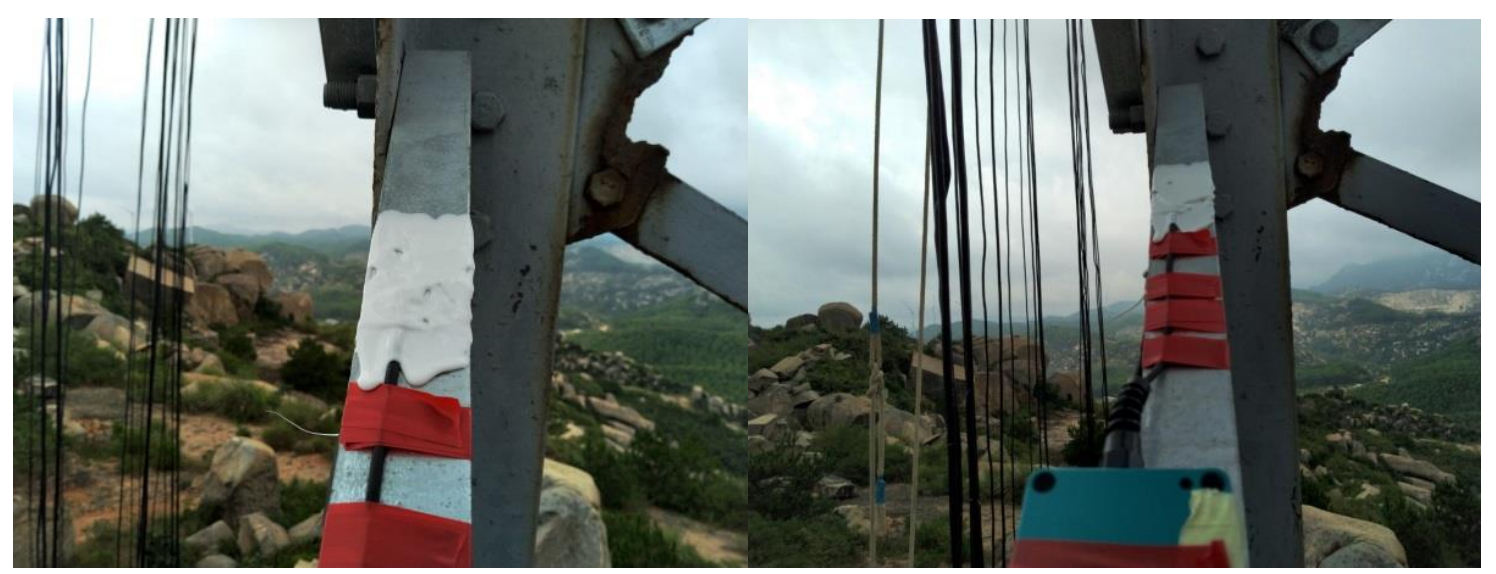

Figure 3. Schematic Diagram of Terrain Altitude within Simulated Range (Unit: m)

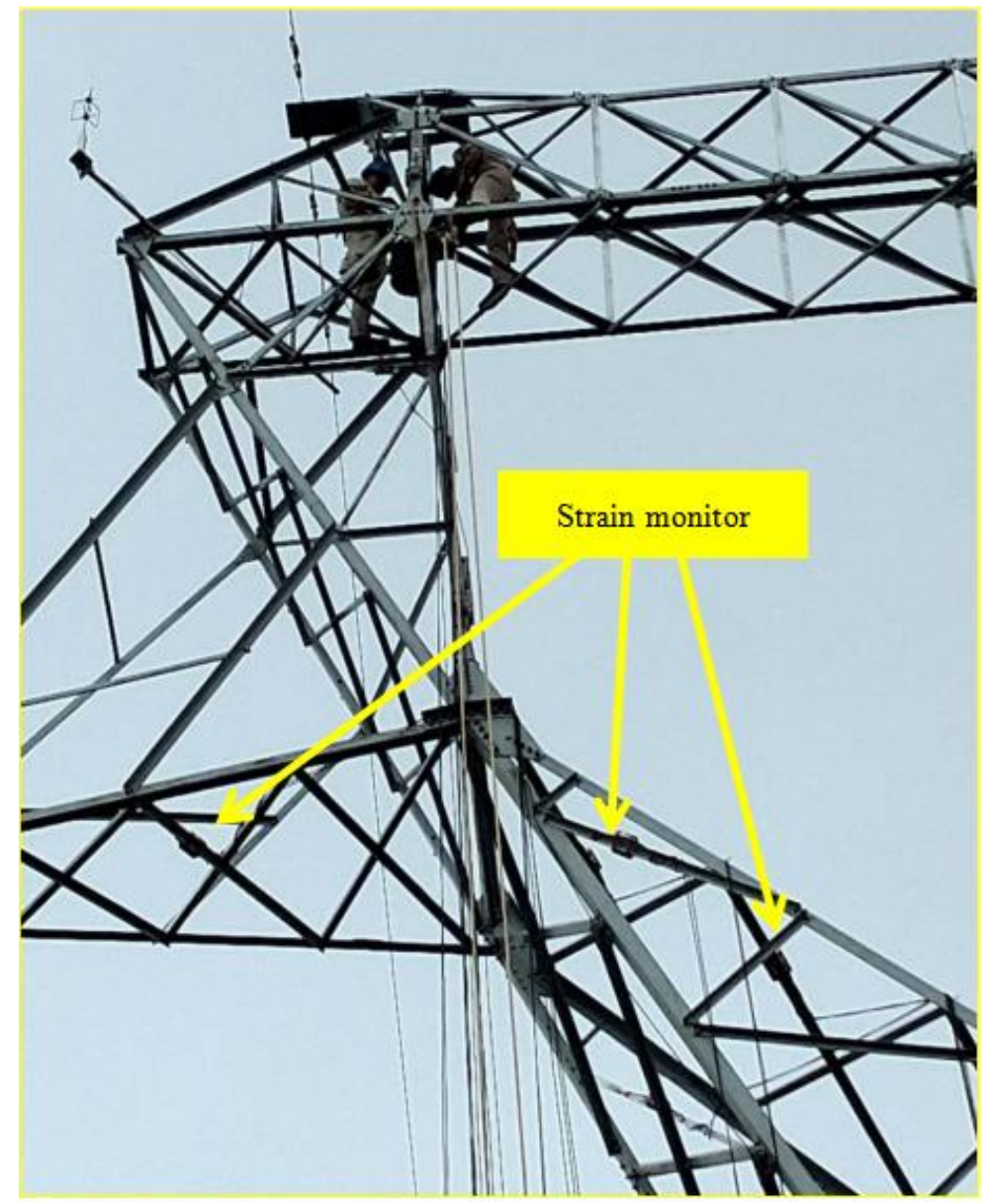

Figure 4. Stain Foil Installed on Tower (Overall)

During the installation process of strain gauge, it is required to conduct operation in strict accordance with the following steps: (1) Member polishing. In the ruled position, polish a square region with dimensions of $15 \mathrm{~mm} * 15 \mathrm{~mm}$ as the measuring point, with the surface reaching a certain smooth degree. (2) Surface cleaning. Adopt absolute ethyl alcohol to clean the surface, make the surface clean without any sundry, and then wait till that the surface is dry completely. (3) Coat primer and paste the strain gauge, and coat a thin layer of B-711 glue solution on the part to be pasted with test piece with make-up pen; next, extrude bubble and the remaining glue solution with finger along with the axial direction of strain gauge, thus removing the thin film of Teflon. (4) Wait till that the primer is dry. (5) Coating AZ-710 protective glue: coat AZ-710 protective glue in the strain gauge zone, with the area 1.5 times greater than the strain gauge, with thickness of about $0.2 \mathrm{~mm}$. (6) Wait till that the glue is dry. (7) Pasting aluminum foil. Paste the aluminum foil to make it cover the strain gauge zone completely. (8) Coating G-704 glue. Coat the G-704 glue on the aluminum foil, and make it cover the edge of aluminum foil completely, with thickness of about $0.3 \mathrm{~mm}$. (9) Fixing conductor and transmitter. Adopt 
adhesive tape to bind the conductor on steel support, and then adopt steel wire for binding in due positions.

\section{Analysis on Strain Monitoring Data}

After the installation of monitoring equipment was completed, the strain acquisition work in 36 strain monitoring points for consecutive 3 months was completed. Table 2 gives the data of monitoring value change of various strain monitoring points during the whole monitoring process. Figure 5 gives the strain change curves of 6 poles whose dynamic stresses are relatively high. During the monitoring period, the scope of the maximum value of one-day strain fluctuation of 36 strain monitoring points was $9.61 \mathrm{MPa}-42.41 \mathrm{MPa}$; the scope of the minimum value of one-day strain fluctuation was $0.21 \mathrm{MPa}-3.47 \mathrm{MPa}$; and the scope of the maximum value of strain fluctuation within the whole period was 11.59MPa-46.53MPa. Assume that the initial stress of pole corresponds to the installation working condition (corresponding to the wind speed of $5 \mathrm{~m} / \mathrm{s}$ ), then the average stresses of 18 pieces of poles under monitoring will be as shown in Table 3 . From Table 3 , it can be known that during this strong breeze process, all iron tower poles were within the scope of limit stress, and there was still a relatively large safety margin, and the overall safety performance of iron tower was good.

Table 2. Change of Monitoring Values of Various Strain Monitoring Points during Whole Monitoring Process

\begin{tabular}{|c|c|c|c|c|c|c|c|}
\hline $\mathrm{S} / \mathrm{N}$ & $\begin{array}{l}\text { Maximum } \\
\text { Value of } \\
\text { One-day } \\
\text { Strain } \\
\text { Fluctuation } \\
\text { Value } / \mu \varepsilon\end{array}$ & $\begin{array}{c}\text { Minimum } \\
\text { Value of } \\
\text { One-day } \\
\text { Strain } \\
\text { Fluctuation }\end{array}$ & $\begin{array}{l}\text { Maximum } \\
\text { Value of } \\
\text { Fluctuation } \\
\text { within the } \\
\text { Strain } \\
\text { Monitoring } \\
\text { Period } \\
\end{array}$ & $\mathrm{S} / \mathrm{N}$. & $\begin{array}{c}\text { Maximum } \\
\text { Value of One- } \\
\text { day Strain } \\
\text { Fluctuation } / \mu \varepsilon\end{array}$ & $\begin{array}{c}\text { Minimum Value } \\
\text { of One-day Strain } \\
\text { Fluctuation } / \mu \varepsilon\end{array}$ & $\begin{array}{c}\text { Maximum Value } \\
\text { of Strain } \\
\text { Fluctuation } \\
\text { within the } \\
\text { Monitoring } \\
\text { Period } / \mu \varepsilon\end{array}$ \\
\hline $1-1$ & 108.91 & 9.75 & 116.57 & $10-1$ & 98.61 & 6.68 & 109.23 \\
\hline $1-2$ & 119.16 & 10.98 & 149.71 & $10-2$ & 118.00 & 10.96 & 154.34 \\
\hline $2-1$ & 88.54 & 11.29 & 103.94 & $11-1$ & 73.61 & 3.81 & 94.33 \\
\hline $2-2$ & 129.39 & 11.06 & 162.78 & $11-2$ & 85.17 & 6.88 & 96.35 \\
\hline $3-1$ & 79.37 & 3.10 & 121.8 & $12-1$ & 65.88 & 6.26 & 67.22 \\
\hline $3-2$ & 78.72 & 6.07 & 93.36 & $12-2$ & 76.72 & 6.26 & 102.45 \\
\hline 4-1 & 86.74 & 4.90 & 91.65 & $13-1$ & 78.98 & 4.66 & 104.12 \\
\hline $4-2$ & 59.52 & 5.48 & 62.56 & $13-2$ & 60.00 & 5.27 & 73.06 \\
\hline $5-1$ & 102.1 & 5.68 & 131.14 & 14-1 & 75.78 & 7.39 & 76.51 \\
\hline $5-2$ & 86.86 & 8.71 & 95.52 & $14-2$ & 109.63 & 5.92 & 131.84 \\
\hline $6-1$ & 56.80 & 3.67 & 74.35 & $15-1$ & 49.83 & 3.95 & 56.25 \\
\hline $6-2$ & 78.29 & 5.48 & 95.58 & $15-2$ & 73.07 & 5.18 & 81.43 \\
\hline $7-1$ & 101.15 & 7.99 & 118.59 & 16-1 & 69.55 & 1.02 & 91.22 \\
\hline $7-2$ & 115.95 & 8.75 & 129.18 & $16-2$ & 46.67 & 2.85 & 101.82 \\
\hline $8-1$ & 77.52 & 9.02 & 90.4 & $17-1$ & 54.67 & 3.23 & 60.62 \\
\hline $8-2$ & 205.86 & 16.84 & 225.89 & $17-2$ & 70.26 & 4.62 & 89.11 \\
\hline $9-1$ & 107.29 & 6.79 & 128.26 & $18-1$ & 90.22 & 4.50 & 96.75 \\
\hline $9-2$ & 122.90 & 10.36 & 149.48 & $18-2$ & 84.18 & 5.29 & 93.99 \\
\hline
\end{tabular}



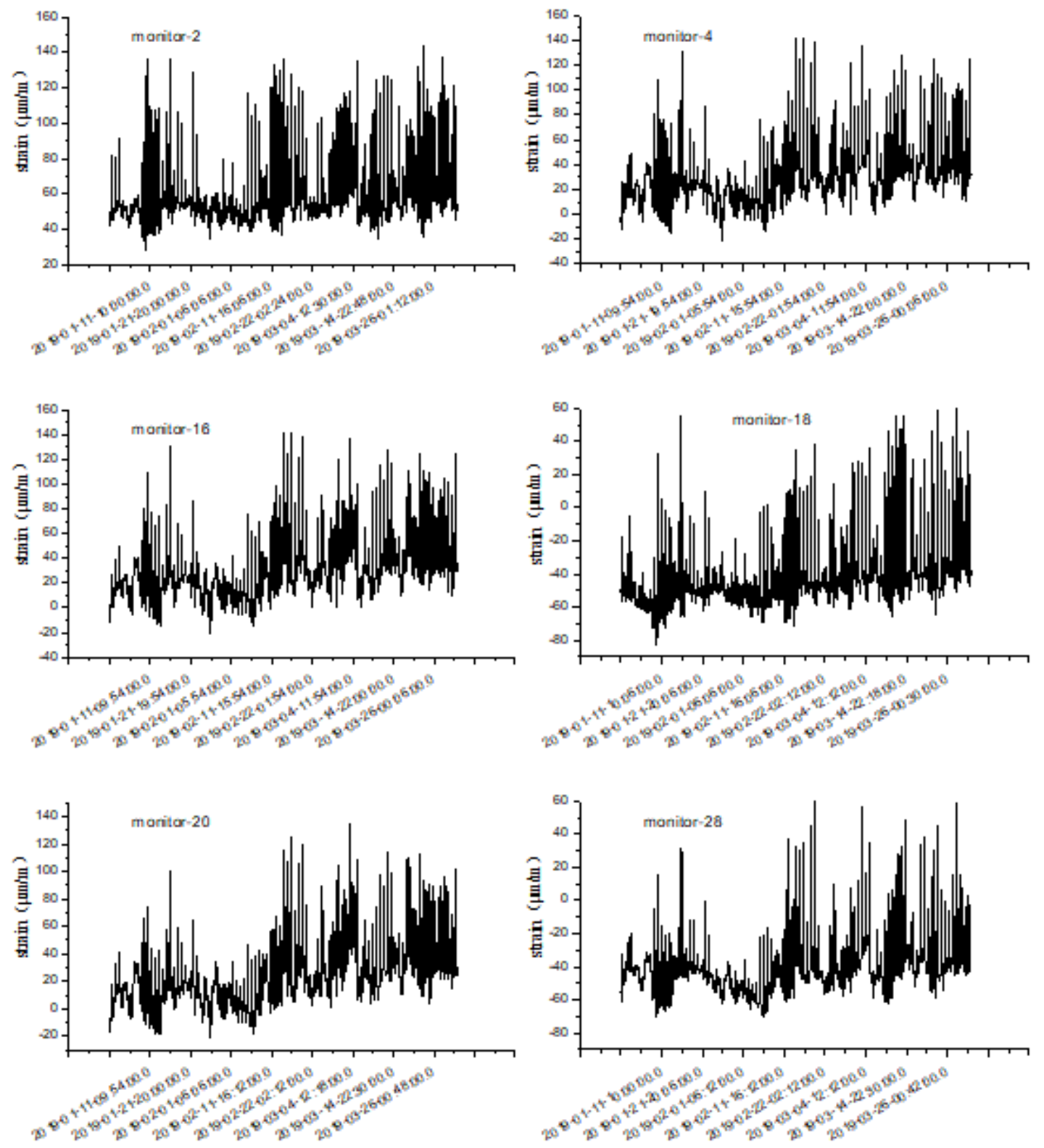

Figure 5. Strain Change Curves of 6 Poles with Relatively High Dynamic Strain

Table 3. Top 18 Poles with Relatively High Stresses

\begin{tabular}{|c|c|c|c|c|c|}
\hline Sorting & Pole No. & $\begin{array}{c}\text { Average Stress } \\
\text { (Mpa) }\end{array}$ & Sorting & Pole No. & $\begin{array}{c}\text { Average Stress } \\
\text { (Mpa) }\end{array}$ \\
\hline 1 & $50-210$ & 94.68 & 10 & $1030-1070$ & 87.03 \\
\hline 2 & $170-372$ & 94.68 & 11 & $670-690$ & 87.03 \\
\hline 3 & $530-690$ & 90.18 & 12 & $870-972$ & 87.03 \\
\hline 4 & $350-390$ & 89.73 & 13 & $690-752$ & 86.13 \\
\hline 5 & $210-350$ & 89.28 & 14 & $10-52$ & 86.13 \\
\hline 6 & $10-50$ & 87.48 & 15 & $210-230$ & 85.68 \\
\hline 7 & $772-870$ & 87.48 & 16 & $1171-1211$ & 85.68 \\
\hline 8 & $690-730$ & 87.48 & 17 & $50-230$ & 85.23 \\
\hline 9 & $970-1032$ & 87.48 & 18 & $750-812$ & 85.23 \\
\hline
\end{tabular}

\section{Conclusion}

In this Project, the iron tower of coastal power transmission line of Fujian Province was adopted as research object, and 36 strain gauges were installed on it.
Through consecutive 3 months of strain monitoring and the stress values of main stress poles corresponding to installation working conditions obtained by combining theoretical analysis, the possible maximum stress of main stress poles of iron tower within the whole monitoring period was obtained. The monitoring result shows that 
within the 3-month monitoring period, the iron tower is in good stress status, with sufficient safety margin.

\section{Acknowledgments}

This work has been funded by the technology project of State Grid Fujian Electric Power Company (Project number: 52130417000T). The authors would like to thank the sponsor of State Grid Fujian Electric Power Company.

\section{References}

1. Wang Jian. Research on Meteorological Disaster Risk Analysis and Fault Early Warning Methods for Overhead Transmission Lines [D]. Chongqing University, 2016.

2. Zhi Lixiang. Application Research of Key Technologies for Transmission Lines Remote Online Monitoring System [D]. China Jiliang University, 2014.

3. Xie Lipeng. Research on On-line Monitoring System of High-voltage Power Transmission Line [D]. Northeast Petroleum University, 2013.

4. Gao Chao. The Development and Application of Network Transmission Lines' Monitoring [D]. North China Electric Power University, 2013.

5. Liao Mingjin. Analysis on Stress of Iron Tower of Power Transmission Line and Research about Online Monitoring Technology [D]. Xi'an Polytechnic University, 2016.

6. Huang Xinbo, Liao Mingjin, Xu Guanhua, Zhu Yongcan, Zhao Long. Stress Monitoring Method Applying FBG Sensor for Transmission Line Towers [J]. Electric Power Automation Equipment, 2016, 36(04): 68-72.

7. Ji Xiangke, Zhang Xuan, Wan Shuting, Liu Ronghai, Zheng Xin. Research on Transmission Tower of Microscopic Stress and Strain Based on FBG Sensor [J]. Yunnan Electric Power, 2016, 44(02):36-38+48.

8. Zhang Xuan. Research about stress Monitoring of Iron Tower Structure Based on Stress Detection Technology [D]. North China Electric Power University, 2016.

9. Zhang Hualin, Yang Yuanhong, Yang Junsheng, He Wei. Application of Sensor in Monitoring Impact of Tunnel Excavation on above Iron Tower [J]. Transducer and Microsystem Technologies, 2011, 30(10):150-152.

10. $\mathrm{Xu}$ Linxiao. Static Design and Optimization of Triangular Transmission Tower Supporting $750 \mathrm{kV}$ Electric Wires [D]. Lanzhou University, 2016.

11. Qiao Jun. Brief Discussion about Pasting Method of Resistance Strain Foil [J]. Technological Pioneers, 2013, 21:119+160.
12. Liu An. Working Principle and Application of Resistance Strain Type Sensor [J]. College Entrance Examination, 2013, 04:83-84. 